\title{
MONOTONICITY OF BUCKLING LOADS UNDER SYMMETRIZATION*
}

\author{
BY \\ SHOSHANA ABRAMOVICH \\ Naval Postgraduate School, Monterey, California**
}

1. Introduction. We will deal with a slender column which is subjected to an axial compressive load $p$ which may cause it to buckle. The governing differential equation for the displacement $w(x)$ from its straight equilibrium position is [2]

$$
\left[E I(x) w^{\prime \prime}(x)\right]^{\prime \prime}+p w^{\prime \prime}(x)=0 .
$$

Here $x$ is the distance measured from one end of the column, $E$ is the modulus of elasticity, and $I(x)=1 / Q(x)$ is the moment of inertia of the cross section of the column about a line passing through its centroid but perpendicular to the plane of buckling. We assume that $E$ is constant but that $I(x)$ is a function of $x$. We will, however, assume that all cross sections are similar. This implies that

$$
I(x)=\frac{1}{Q(x)}=K A^{2}(x)
$$

where $A(x)$ is the area of the cross section and $K$ is a constant which depends only on the particular shape of the cross section. The volume of the column is given by

$$
V=\int_{-1}^{1} A(x) d x .
$$

The boundary conditions we will consider in connection with the column are of the following form:

$$
\begin{gathered}
\text { Pinned at } x=-1 \text { and at } x=1: \\
w(-1)=w^{\prime \prime}(-1)=w(1)=w^{\prime \prime}(1)=0, \\
\text { clamped at } x=-1 \text { and free at } x=1 \text { : } \\
w(-1)=w^{\prime}(-1)=E I(1) w^{\prime \prime}(1)=p w^{\prime}(1)-\left(E I(1) w^{\prime \prime}(1)\right)=0, \\
\text { and clamped at } x=-1 \text { and } x=1 \text { : } \\
w(-1)=w^{\prime}(-1)=w(1)=w^{\prime \prime}(1)=0 .
\end{gathered}
$$

* Received January 31, 1984.

** The author's permanent address is the Department of Mathematics, Haifa, Israel. 
Besides the problem of the column we will deal with the problem of the vibrating string. Let $Q(x) \geqslant 0,-1 \leqslant x \leqslant 1$, be the density of a string of unit tension. The frequencies of the string are determined by the eigenvalues of the differential equation

$$
y^{\prime \prime}(x)+\lambda Q(x) y(x)=0
$$

with various boundary conditions. The boundary conditions we will consider in connection with (7) are of the form

$$
\begin{aligned}
& y(-1)=y(1)=0, \\
& y^{\prime}(-1)=y(1)=0,
\end{aligned}
$$

and

$$
\begin{gathered}
y^{\prime}(-1)-A y(-1)=y^{\prime}(1)+B y(1)=0, \\
A \leqslant B, \quad y^{\prime}(-1) y(-1)-y(1) y^{\prime}(1)+\int_{-1}^{1} y^{\prime 2} d x>0 .
\end{gathered}
$$

The main object of this paper is to show the monotonicity of frequencies of a string under a symmetrization method applied to $Q(x)$.

Following [7], we introduce in the case of the column the bending moment $y(x)$ defined by

$$
y(x)=-E I(x) w^{\prime \prime}(x)
$$

Equation (1) becomes

$$
y^{\prime \prime}=P w^{\prime \prime}
$$

Using (11), we obtain (7), where

$$
P=\lambda E K, \quad Q(x)=\frac{1}{A^{2}(x)} .
$$

Integrating (12) twice from -1 to $x$, we find after setting $x=1$ that

$$
y^{\prime}(1)-y^{\prime}(-1)=P\left[w^{\prime}(1)-w^{\prime}(-1)\right]
$$

and

$$
y(1)-y(-1)-2 y^{\prime}(-1)=P\left[w(1)-w(-1)-2 w^{\prime}(-1)\right] .
$$

Combining (14) and (15) with (4) and (5), we get equivalent boundary conditions on $y(x)$ in the following cases:

$$
\begin{gathered}
\text { Pinned at } x=-1 \text { and } x=1: \quad y(-1)=y(1)=0, \\
\text { clamped at } x=-1 \text { and free at } x=1: \quad y^{\prime}(-1)=y(1)=0,
\end{gathered}
$$

and

$$
\begin{gathered}
\text { clamped at } x=-1 \text { and } x=1: \\
y^{\prime}(-1)=y^{\prime}(1), \quad y(1)-y(-1)=2 y^{\prime}(1) .
\end{gathered}
$$

The differential equation (7) with each of the boundary conditions (8), (9), (9.1), or (10) form self-adjoint eigenvalue problems for determination of $\lambda$ [which then determines $P$ by (13)] [3]; therefore, there will be an infinite sequence of eigenvalues $\lambda_{n} \geqslant 0$. The critical buckling load $P$ will be determined by using the smallest positive eigenvalue. $\lambda=0$ is not 
an eigenvalue of (7) subject either to (8), (9), or (10), and as $P=0$ is never an eigenvalue of (1) subject to any of the boundary conditions (4), (5), or (6), therefore in these cases the critical buckling load is determined by using (13) and the first eigenvalue. $\lambda=0$ is an eigenvalue of multiplicity 2 of the system (7) and (9.1); therefore, the critical buckling load must be determined by using (13) and the third eigenvalue, say $\lambda_{3}$, of the system (7) and (9.1). In all cases the eigenfunctions $y(x)$ and $w(x)$ are related by

$$
y(x)-y(-1)-(x+1) y^{\prime}(-1)=P\left[w(x)-w(-1)-(x+1) y^{\prime}(-1)\right] .
$$

2. Symmetrization method. The main object of this paper is to show monotonicity of the buckling load of a column and monotonicity of frequencies of a vibrating string under the symmetrization method applied to $A(x)$ or $Q(x)$.

A function $Q(x)$ in $[-1,1]$ is called left-balanced if for any $x \in[0,1], Q(-x) \geqslant Q(x)$ [1].

A positive function $Q(x)$ is said to be of class $A$ in $[-1,1]$ if $Q(x) \in C$ is left-balanced, nonincreasing in $[-1, l]$, and nondecreasing in $[l, 1]$ for some $l,-1<l \leqslant 1$. For a function $Q(x)$ belonging to class $A$ we denote the inverse function of $Q(x)$ by $x_{1}(y)$ for $x \in[-1, l]$ and by $x_{2}(y)$ for $x \in[l, 1]$.

We define a class of functions $Q(x, a),-1 \leqslant a \leqslant 1,-1 \leqslant x \leqslant 1$, by a procedure called continuous symmetrization.

For $0 \leqslant a \leqslant \frac{1}{2}$ and $x \in[-1,2 a l]$ we denote the inverse function of $Q(x, a)$ by $x_{1}(y, a)$, and for $x \in[2 a l, 1]$ we denote the inverse function by $x_{2}(y, a)[5$, p. 200]:

$$
\begin{aligned}
& x_{1}(y, a)=\left(a+\frac{1}{2}\right) x_{1}(y)+\left(a-\frac{1}{2}\right) x_{2}(y), \\
& x_{2}(y, a)=\left(a+\frac{1}{2}\right) x_{2}(y)+\left(a-\frac{1}{2}\right) x_{1}(y) .
\end{aligned}
$$

For $\frac{1}{2} \leqslant a \leqslant 1$ and $x \in[-1, l(2-2 a)+2 a-1]$ we denote the inverse function of $Q(x, a)$ by $x_{1}(y, a)$ and for $x \in[l(2-2 a)+2 a-1,1]$ we denote the inverse function by $x_{2}(y, a)$ :

$$
\begin{aligned}
& x_{1}(y, a)=x_{1}(y)+(2 a-1)\left(1-x_{2}(y)\right), \\
& x_{2}(y, a)=x_{2}(y)+(2 a-1)\left(1-x_{2}(y)\right) .
\end{aligned}
$$

For $-1 \leqslant a \leqslant 0$ and $-1 \leqslant x \leqslant 1$ we denote

$$
\begin{aligned}
& x_{1}(y, a)=-x_{2}(y,-a), \\
& x_{2}(y, a)=-x_{1}(y,-a) .
\end{aligned}
$$

If $Q(-1)>Q(1)$ we enlarge the interval of deînition of $x_{2}(y)$ by adding the interval $Q(1) \leqslant y \leqslant Q(-1)$, on which we define $x_{2}(y)=1$. Obviously, we have $Q\left(x, \frac{1}{2}\right)=Q(x)$; $Q(x, 1)$ is the decreasing rearrangement; $Q(x, 0)$ is the symmetrical increasing rearrangement; and $Q(x,-1)$ is the increasing rearrangement of $Q(x)[4,6]$ (see Fig. 1).

The functions $Q(x, a)$ are equimeasurable; i.e., for each $y, m(x, Q(x, a) \geqslant y,-1 \leqslant x$ $\leqslant 1)=m(x, Q(x) \geqslant y,-1 \leqslant x \leqslant 1)[4]$.

REMARK. In the case that the graph of $Q(x)$ is a polygon, the definition of $Q(x, a)$ for $\frac{1}{2} \leqslant a \leqslant 1$ coincides with the definition of $Q(x, a)$ for $0 \leqslant a \leqslant \frac{1}{2}$; otherwise we cannot use the same definition for all $0 \leqslant a \leqslant 1$ because the graph we get might describe a non-single-valued function for $\frac{1}{2} \leqslant a \leqslant 1$. 
624

SHOSHANA ABRAMOVICH
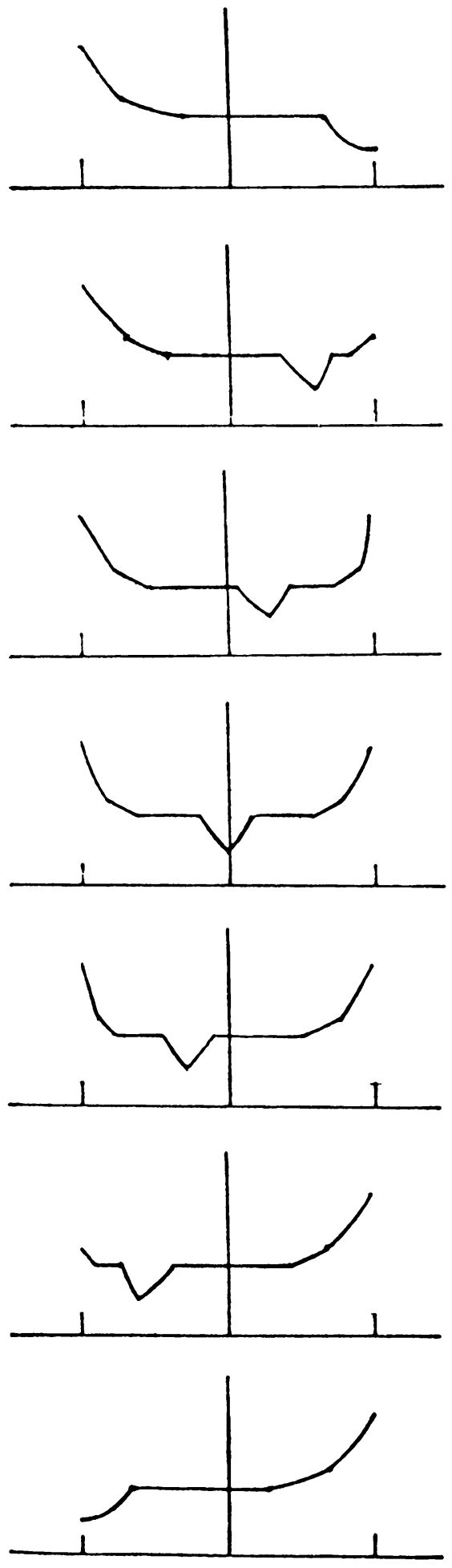

$Q(x, 1)$ the decreasing rearrangement of $Q(x)$

$Q(x)=Q(x, 1 / 2), Q(x)$ is the given function

$Q(x, 1 / 4)$

$Q(x, 0)=Q^{+}(x)$ the symmetrical increasing rearrangement

$Q(x,-1 / 4)$

$Q(x,-1 / 2)$

$Q(x,-1)$ the increasing rearrangement of $Q(x)$

FIG. 1. 
3. Results. The same proofs as in [1] lead to the following theorems.

THEOREM 1 . Let $Q(x) \geqslant 0$ be of class $A$. Then $\lambda_{1}(a)$, the first eigenvalue of the systems

$$
\left.\begin{array}{c}
y^{\prime \prime}(x)+\lambda(a) Q(x, a) y(x)=0, \quad y( \pm 1)=0, \\
y^{\prime \prime}(x)+\lambda(a) Q(x, a) y(x)=0, \quad y^{\prime}(-1)=y(1)=0, \\
y^{\prime \prime}(x)+\lambda(a) Q(x, a) y(x)=0, \\
y^{\prime}(-1)-A y(-1)=y^{\prime}(1)+B y(1)=0, \\
y^{\prime}(-1) y(-1)-y(1) y^{\prime}(1)+\int_{-1}^{1} y^{\prime 2} d x>0, \\
A \leqslant B,
\end{array}\right\}
$$

decreases with $a, 0 \leqslant a \leqslant 1$.

TheOREM 2. Under the same assumptions as in Theorem $1, \lambda_{1}(a)$, the first eigenvalue of the system $(9 \mathrm{a})$, decreases with $a,-1 \leqslant a \leqslant 1$, and the same holds under the additional assumption $A \leqslant 0$ for the first eigenvalue of (10a).

From Theorems 1 and 2, and using (12) and (13), we get

THEOREM 3. The buckling load $P(\alpha)$ of a column pinned at both ends and having area function $A(x)=1 / \sqrt{Q(x)}$, where $Q(x)$ is of class $\mathrm{A}$, decreases with $a, 0 \leqslant a \leqslant 1$.

Theorem 4. The buckling load $P(\alpha)$ of a column free at $x=1$ and clamped at $x=-1$, having area functions $A(x)=1 / \sqrt{Q(x)}$, where $Q(x)$ is of class A, decreases with $a$, $-1 \leqslant a \leqslant 1$.

THEOREM 5. Let $Q(x)$ be of class A in $-\frac{1}{2} \leqslant x \leqslant 0$, and let

$$
Q^{*}(x)=\left\{\begin{array}{l}
Q(-1-x), \quad-1 \leqslant x \leqslant-\frac{1}{2}, \\
Q(-x), \quad 0 \leqslant x \leqslant 1
\end{array}\right.
$$

Then the buckling load of a column clamped at both ends -1 and 1 , having area function $A^{*}(x, a)=1 / Q^{*}(x, a)$, increases with $a,-1 \leqslant a \leqslant 1$, when continuous symmetrization is applied to $Q(x)$.

For the proof consider the new eigenvalue problem:

$$
\text { (*) } \quad Z^{\prime \prime}+\mu Q^{*}(x, a) Z=0, \quad Z^{\prime}(-1)=Z^{\prime}(1)=0 .
$$

Now for this new problem, the symmetry of $Q^{*}(x, a)$ does imply that its third eigenfunction has even symmetry about $x=0$ and odd symmetry about $x=-1 / 2$ and $x=+1 / 2$. Therefore $Z_{3}(-1)=Z_{3}(1)$ and $Z_{3}(x)$ satisfies the boundary conditions for a clamped column. Therefore $Z_{3}(x)$ is also an eigenfunction of (7) and (9.1). Thus $\mu_{3}(a)=\lambda_{3}(a)$, and the third eigenfunction of $(*)$ above can also be taken to be the third eigenfunction of (7) and (9.1).

One may thus select the third eigenfunction $Y_{3}(x)$ so that it satisfies the conditions

$$
Y^{\prime}(-1)=Y(-1 / 2)=Y^{\prime}(0)=Y(1 / 2)=Y^{\prime}(1)=0
$$

(although they may not be true for both eigenfunctions).

This allows us to consider the clamped symmetric column as being composed of four distinct congruent columns, each of which is clamped at one end. Then we use Theorem 2 for each of the intervals $\left[-1,-\frac{1}{2}\right],\left[-\frac{1}{2}, 0\right],\left[0, \frac{1}{2}\right],\left[\frac{1}{2}, 1\right]$, and the proof of Theorem 5 is complete (see Fig. 2). 


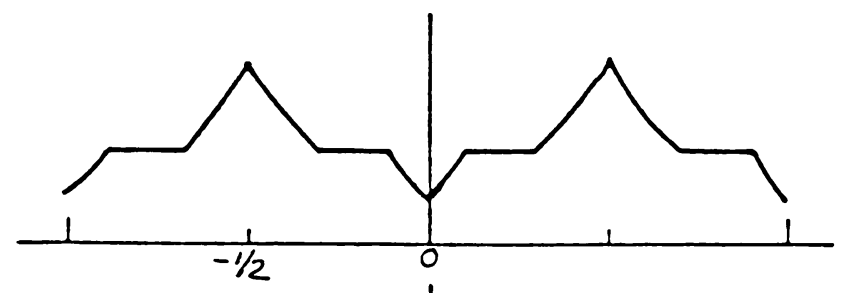

The shape of the strongest column $Q^{*}(x, 1)$ the decreasing rearrangement of $Q(x)$ in $[-1 / 2,0]$ $Q(x, 1)=Q_{-2}(x)$ (see [2])

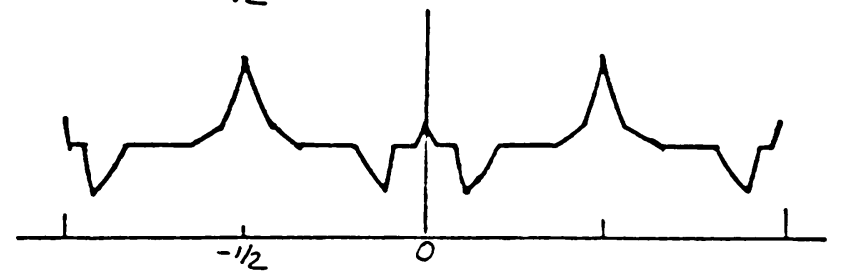

$Q(x)=Q^{*}(x, 1 / 2)$

$Q(x)$ is the given column

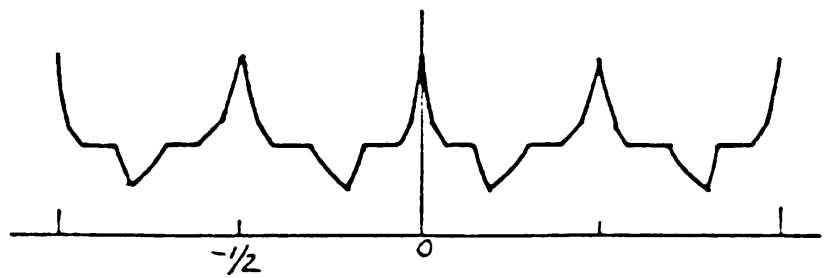

$Q^{*}(x, 1 / 4)$

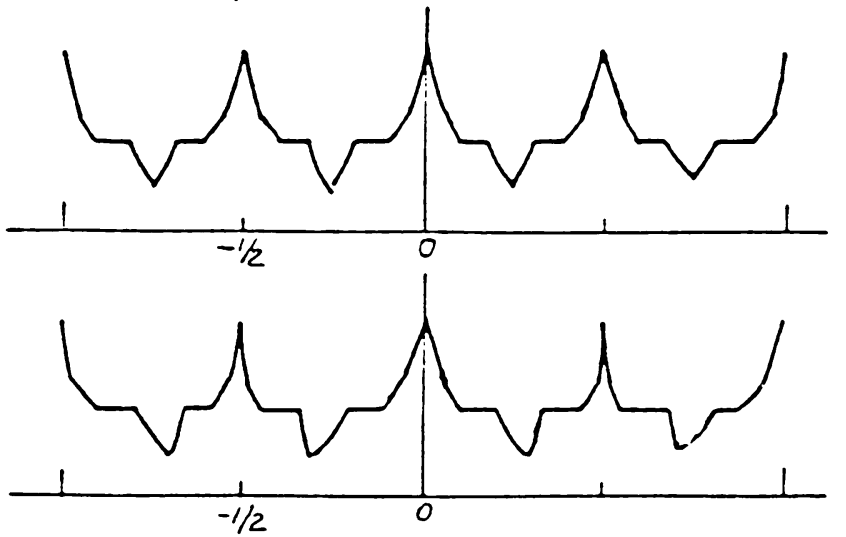

$Q^{*}(x, 0)=Q_{+4}^{*}(x)$ (see [2])

$Q^{*}(x, 0)$ is the symmetrical increasing rearrangement of $Q(x)$ in $[-1 / 2,0]$

$Q^{*}(x,-1 / 4)$

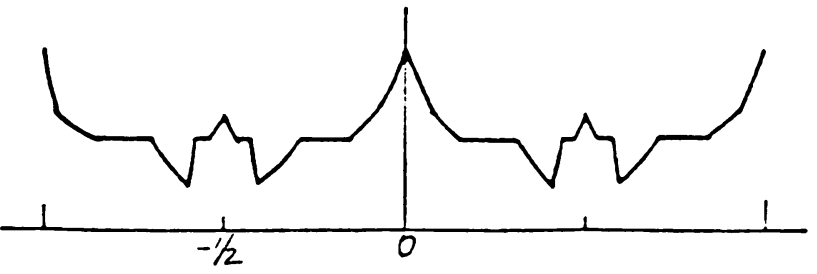

$Q^{*}(x,-1 / 2)$

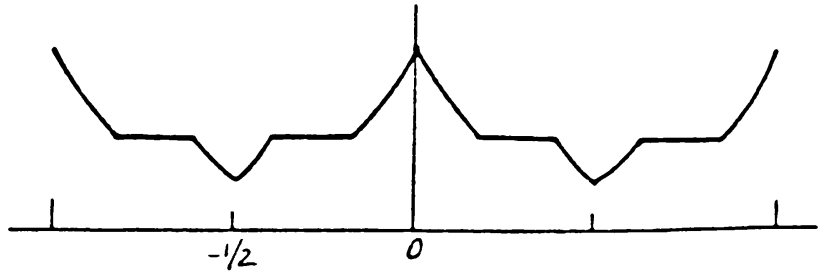

The shape of the weakest column $Q^{*}(x,-1)$ the increasing rearrangement of $Q(x)$ in $[-1 / 2,0]$ $Q^{*}(x,-1)=Q_{+2}^{*}(x)($ see $[2])$

FIG. 2 
In this last theorem we see that not only is the buckling load of $Q(x)$ between the buckling loads of $Q_{-2}^{*}(x)=Q^{*}(x, 1), Q_{+2}^{*}(x)=Q^{*}(x,-1)$ as proved in [2], but also under the conditions of the theorem there is a monotonicity phenomenon of the buckling load between these extremities.

\section{REFERENCES}

[1] S. Abramovich, Monotonicity of eigenvalues under symmetrization, SIAM J. Appl. Math. 28, 350-360 (1975)

[2] D. C. Barnes, Buckling of columns and rearrangement of functions, Quart. Appl. Math. 41, 169-180 (1983)

[3] R. Courant and D. Hilbert, Methods of mathematical physics, Interscience, New York, 1953

[4] G. H. Hardy, J. E. Littlewood, and G. Pólya, Inequalities, Cambridge Univ. Press, Cambridge, 1964

[5] G. Pólya and G. Szegö, Isoperimetric inequalities in mathematical physics, Annals of Mathematics Studies, no. 27, Princeton Univ. Press, Princeton, N. J. (1951)

[6] B. Schwarz, On the extrema of frequencies of nonhomogeneous strings with equimeasurable density, J. Math. Mech. 10, 401-422 (1961)

[7] I. Tadjbakhsh and J. B. Keller, Strongest columns and isoperimetric inequalities for eigenvalues, J. Appl. Mech. 29, $159(1962)$ 